\title{
Lumen
}

Selected Proceedings from the Canadian Society for Eighteenth-Century Studies

\section{Scènes de mariage et populationnisme dans le Supplément au voyage de Bougainville}

\section{Servanne Woodward}

Volume 16, 1997

Freedom and Boundaries

Émancipation et frontières

URI : https://id.erudit.org/iderudit/1012449ar

DOI : https://doi.org/10.7202/1012449ar

Aller au sommaire du numéro

Éditeur(s)

Canadian Society for Eighteenth-Century Studies / Société canadienne d'étude du dix-huitième siècle

ISSN

1209-3696 (imprimé)

1927-8284 (numérique)

Découvrir la revue

Citer cet article

Woodward, S. (1997). Scènes de mariage et populationnisme dans le Supplément au voyage de Bougainville. Lumen, 16, 197-209.

https://doi.org/10.7202/1012449ar d'utilisation que vous pouvez consulter en ligne. 


\section{Scènes de mariage et populationnisme dans le Supplément au voyage de Bougainville}

L'ignorance de nos vices et la naïveté de leurs moeurs les jetèrent dans les bras de leurs lâches ennemis.

Grafigny, «Introduction historique» 253

L'introduction des Lettres d'une Péruvienne exprime quelque indignation à l'endroit du procédé des Espagnols engagés dans la conquête coloniale. D'un roman à l'autre, d'un pays conquis à l'autre, il existe un discours étrangement conforme sur la première rencontre des colonisateurs et des colonisés, au sein de la littérature du dix-huitième siècle français. Le vocabulaire choisi évoque un contrat inégal alliant l'innocence vitale à la destruction programmatique. L'amitié, l'amour spontané, sont confrontés à l'agression avide et consommatrice. On finit par distinguer les variations multiples du scénario d'une liaison dangereuse entre les colonisés et les colonisateurs, qui est à l'origine d'un mythe de fondation nationale. Il crée pour l'Espagne et pour la France une identité coloniale appelée à «féconder» les populations étrangères - à s'y substituer. De paternité française, la génération future des colonies sera soit malade (morte, syphilitique), soit conquérante, en ce que les marins français (qui auront procréé à la place des Tahitiens) seront implantés sur l'île par le biais de leur progéniture. Ces enfants de Français seront même adoptés par des pères tahitiens. La prospérité coloniale, c'est alors le détour de tous les moyens de production et de reproduction des autochtones au profit de la population colonisatrice. Ce type de relation implique l'utilisation de la femme tahitienne (de son ventre) comme instrument de colonisation. 


\section{Promesses}

Diderot engage ses propres fantasmes vis-à-vis du mariage et des femmes dans le Supplément. Rappelons que Diderot a épousé Antoinette Champion parce qu'il l'avait mise enceinte. La reproduction précède le mariage dans le Supplément, mais le père n'a pas besoin d'élever son enfant dans cette terre qui n'est pas chrétienne. Si le prêtre du Supplément copule librement avec deux jeunes filles (en bénéficiant de l'approbation de leur père), c'est qu'elles pourront ainsi être mariées à des jeunes gens locaux rassurés sur la fertilité de leurs épouses. Mais ainsi, la descendance de paternité française se substitue à celle des Tahitiens. Remarquons encore au niveau du fantasme que Diderot avait cherché à adopter légalement comme son «fils» celui qui était déjà devenu son beau-fils.

Ces méthodes de parenté substitutives commencent dans le Supplément par la voix tahitienne - voix d'expression, de protestation. En premier lieu, Diderot prête voix aux Tahitiens par le personnage d'Orou. Edward W. Said nous invite à suivre une méthodologie qui identifie la position de l'auteur comme «lieu stratégique» en relation au pouvoir référentiel de son texte, mais aussi en tant que prise de position vis-à-vis des deux camps, colonisateur et colonisé (Orientalism 20). ${ }^{1}$ D'une perspective française, le vieillard dit tout ce qu'il y a à dire sur la colonisation. Il a fait son procès. Et puis Orou vient le compléter, ajoutant des arguments qui annulent finalement le discours du vieillard. Orou remplace donc ce dernier en proposant l'apologie de l'apport des colonisateurs aux colonisés. La thèse est suivie d'une antithèse, puis d'un atermoiement qui se résume finalement à une prise de position pleinement coloniale. Diderot est rarement aussi clair dans son art dialogique qui joue plus souvent sur l'indétermination philosophique. C'est que nous sommes peut-être plus près de l'ordre politique.

Dans Le concentrique et l'excentrique, Georges Benrekassa s'interroge sur ce qui permet à Diderot de tenir «effectivement plusieurs discours à la fois» à l'occasion du Supplément (217). Mais ces énoncés divergents et contradictoires sont résolus par leur ordre d'apparition. La polyphonie du Supplément est "poétique du déguisement» conclut Marie-Hélène Chabut, qui détaille les contradictions travaillant le discours d'Orou, dans lequel un pluralisme apparent se ramène finalement à la parole de l'aumônier. ${ }^{2}$ Ce dernier partage le point de vue de Diderot qui aurait peut-être endossé le surplis de son frère pour l'occasion. A travers le discours d'Orou, la «Nature» exprime la culture française, stoïcochrétienne, adoucie d'une tolérance philosophique (une synthèse composite des deux frères Diderot). Diderot cherche à démontrer que la nature rappelle nécessairement la culture parce que l'une découle de l'autre, inévitablement, nécessairement, et avec la collaboration totale de 
l'une et l'autre. Mieux, séduite par la nature, la culture devra céder pour le bien du progrès, aux avances de la nature qui demande à se faire «posséder.» La nécessité de déguisement n'est pas seulement stratégique; elle vient de la distance culturelle, linguistique, géographique, qui sépare la France de ses colonies.

\section{Redresser/justifier ses torts}

$C^{\prime}$ est qu'Orou parle ailleurs, là où sa voix porte, et il ne peut atteindre les côtes françaises sans truchement. Finalement, cette voix qui pousse en terre étrangère est «exotique» selon la définition encyclopédique, qui relie ce terme exclusivement au jardinage (Enc. VI: 274). La voix tahitienne est géographiquement enracinée. Orou ne peut communiquer en dehors d'une certaine aire audible. Quand son discours est entériné par une écriture double, franco-espagnole, sa voix et son être ont été «naturalisés» dans tous les sens du terme, qui relève aussi de l'acclimatation et de la taxidermie. Tahiti n'est déjà plus la même au premier contact, à ses premières noces illégitimes. Elle est donc inaccessible et sa translation (comme traduction et transport des restes, d'un corps) irréversible.

Le Tahitien ne respire ni ne parle que localement, chez lui. Il est en somme aussi périssable que ces curiosités que l'on cherche à importer toutes vives:

C'est dans ce voyage que l'on a trouvé le quadrupède de dix-sept pieds de hauteur, dont j'ai remis le dessin à $\mathrm{M}$. de Buffon; c'était une femelle qui allaitait un faon dont la hauteur n'était encore que de sept pieds. On tua la mère, le faon fut pris vivant, mais il mourut après quelques jours de marche. M. de Buffon m'a assuré que cet animal est celui que les naturalistes nomment la giroffe. On n'en avait pas revu depuis celui qui fut apporté à Rome du temps de César, et montré à l'amphithéâtre. On a aussi trouvé il y a trois ans, et apporté au Cap, où il n'a vécu que deux mois, un quadrupède d'une grande beauté, lequel tient du taureau, du cheval et du cerf, et dont le genre est absolument nouveau. . . Ce n'est pas sans raison que l'Afrique a été nommée la mère des monstres. (Bougainville, Voyage 428-429)

Constitués d'un mélange d'êtres européens, ces «monstres» sont prompts à se désagréger. Du pingouin apprivoisé au girafeau, tous meurent de faim, tous devaient être «transportés» en Europe (Bougainville, Voyage 102). Leur extrême fragilité et la scopie qu'ils suggèrent, s'inscrivent dans l'espace sacrificiel du théâtre des conquêtes, de l'histoire naturelle et coloniale. ${ }^{3}$ La fragilité de ces êtres composites consacre 
leur non-viabilité et, paradoxalement, justifie leur massacre et exonère la culpabilité des colonisateurs.

Benrekassa observe que les êtres dits «sauvages» "se corrompent dès que touchés» soit par la contagion syphilitique, soit, rajoutons-le, par ce contact que les oiseaux initient, dans les îles encore étrangères à l'homme, où ils viennent curieusement se poser dans les mains de leurs meurtriers, à cause d'une innocence funeste (Concentrique 219; Bougainville, Voyage 81, 99). Dans tous les cas, l'aspect spectaculaire de la rencontre est évoqué, que les animaux se soient approchés par curiosité pour voir les premiers hommes qu'ils aient jamais rencontrés, ou que les Tahitiennes s'ébattent avec les marins de Bougainville sous l'applaudissement public qui consacre cette activité, car l'hospitalité tahitienne demande que les femmes se donnent en public aux nouveaux arrivés. Quoi qu'il en soit, cette Afrique qui combine les animaux d'Europe, ces îles fantastiques où les animaux viennent en masse se laisser voir, toucher, prendre, tuer, cet Otaïti copulant avec les étrangers sains ou syphilitiques, sont destinés à la conquête. Cette dernière peut être française ou romaine. Car l'Encyclopédie de d'Alembert et Diderot définit $\mathrm{d}^{\prime}$ une seule haleine la colonisation française et romaine.

L'être naturel meurt le temps du spectacle. «Morituri te salutant»: "Ceux qui vont mourir vous saluent,» disaient les gladiateurs romains, parlant d'eux-mêmes à la troisième personne, consentant à devenir l'objet d'un spectacle mortel. Prisonniers de guerre, ou bien voués à la servitude volontaire, les gladiateurs sont conviés au duel pour des spectacles originellement commémoratifs "aux pompes funebres des consuls \& des premiers magistrats de la république» (Enc. VII: 694). Les Tahitiennes se livrent à des joutes amoureuses non moins mortelles puisqu'elles contractent la mort par les marins syphilitiques. Orou parlerait pour le monde sauvage qui, selon Benrekassa, "consent à sa propre mort» dans le Supplément (Concentrique 219), comme à Rome les gladiateurs, qui attendent le dernier mot de César, puis de l'opinion publique - le sceau du destin. Il n'est pas vraiment question qu'un coup de culpabilité ou d'attendrissement viennent toucher les nations des vainqueurs. Ces derniers sont simplement conviés au théâtre de leur conquête.

Tout au plus, leur participation scellera le destin. Ce dernier est lié au signe visuel, à un coup de pouce ou au travail de la main formant les caractères d'un système d'échange entre "Nature» et «Culture». La représentation, c'est finalement une traduction mortifère, désagrégeant le corps vivant et le souffle. Il ne reste plus qu'à ce qu'une technique de conservation objectifie les restes d'un corps étranger - un lambeau d'épiderme. 


\section{Unir les destins}

Les représentants du pouvoir politique (Bougainville), ou culturel (Diderot) récupèrent l'expression «sauvage» ou celle de la «Nature»:

.. l'éminente dignité du sauvage est ici niée en même temps qu'affirmée. . . . Bougainville part au milieu du silence; mais il est entendu que ce silence, même réprobateur, lui laisse le dernier mot; et surtout . . . à Diderot . . . par une usurpation nécessaire et inéluctable. (Benrekassa 220)

L'appropriation du destin de l'être colonisé, de sa parole, de son engeance, de sa survie marque les étapes d'un alignement de la «nature» sur le progrès «naturel» - et par là même «inéluctable».

Le Tahiti diderotien est cet Éden mythique au moment du passage au fil de l'écriture "civilisatrice», ou bien cet espace désigné au jeu du destin. L'écriture, la philosophie, l'intellect, les Lumières, entérinent la conquête militaire, ou diplomatique, ou sexuelle, en la justifiant. Derrida constate (de manière très provocatrice) qu'une pensée «hégelienne» confondrait le destin global à celui de l'Europe, identifiable encore récemment écritil, lorsqu'à l'occasion de la guerre "du golfe» un porte-parole de la Maison Blanche aurait déclaré «la fin-de-l'histoire», c'est-à-dire le temps où les «États-nations» tendent unanimement à suivre un modèle identique de «démocraties libérales . . . là où le capital est à la pointe du progrès» (L'autre cap 35-36). Une tradition politique et philosophique se joindrait à une économie coloniale. La fin de l'histoire ce serait pour Tahiti de devenir ce vers quoi elle progresse à son insu depuis toujours: la France. On pourrait parler d'une histoire doublement prégnante: Tahiti doit devenir la France (elle est l'ancêtre naturel de la France, elle est la France à ses origines dans son enfance). Donc Tahiti est la mère de la France (comme la «nature» enfante «la civilisation»). Mais Tahiti est aussi l'enfant, l'héritière de la France, qui à son tour lui donnera les moyens de devenir française.

Ainsi on peut observer les effets de l'étreinte décriée par Grafigny en exergue de ce texte: "L'ignorance de nos vices et la naïveté de leurs moeurs les jetèrent dans les bras de leurs lâches ennemis» (253). Elle parlait de la conquête du Pérou par les Espagnols. Mais les interactions sont identiques à Tahiti, sous l'effet combiné de la colonisation espagnole et française. Si la compagne des marins français ne meurt pas de maladie vénérienne, son commerce avec l'ennemi défait la coutume locale. Les moeurs et le fondement communautaire de la société tahitienne sont changés. Le Supplément oppose l'amour incestueux et «naturel» des Tahitiens à la passion exclusive et recluse importée par les marins de Bougainville. Il ne s'agirait pas tant d'attacher «des idées 
morales» que politiques au gouvernement sexuel français qui ressemble à celui du sérail, selon la description qu'en fait Montesquieu. ${ }^{4}$ De plus, l'inclination réciproque $d u$ frère et de la soeur est opportunément interdite pour que se rétablisse un ordre civil: Merle Louis Perkins rappelle que l'inceste est dangereux pour la royauté, car il pourrait concentrer le pouvoir au sein de cinquante familles pour toute la France (417). L'introduction de la couronne de France dans l'île passe par un alignement du comportement sexuel tahitien sur celui de la famille française: la passion, le secret, l'amour exclusif, la séclusion, la vengeance à l'européenne se sont déjà introduits dans les coeurs de certains et portent quelques ressemblances avec l'amour de sérail par lequel Montesquieu désignait peut-être la nation française plutôt que persane. ${ }^{5}$ La nature est immédiatement sensible aux attouchements de la civilisation, si Tahiti définit l'Europe au premier contact comme héritière et légataire. En conséquence, l'île est immédiatement aliénée, annexée, ou promptement défunte.

\section{Où il est question de natalisme et du mariage}

Orou comprend l'Europe par les lois et la moralité qui régissent le contact sexuel. Elles s'avèrent liées au natalisme cher à Diderot. Antoine Adam a identifié la moralité de "paternité» comme essentielle au Tahiti diderotien (25). Orou a offert sa femme et ses filles à l'aumônier pour tirer de lui une progéniture «enrichissante» - il poursuit en concluant sur l'économie de son offre apparemment gratuite:

Va où tu voudras; et tu trouveras presque toujours l'homme aussi fin que toi. Il ne te donnera jamais que ce qui lui est bon à rien, et te demandera toujours ce qui lui est utile. S'il te présente un morceau d'or pour un morceau de fer, c'est qu'il ne fait aucun cas de l'or, et qu'il prise le fer. (Supplément 175)

Miss Polly Baker avait précédé Orou dans cette considération sur la productivité du mariage aussi «librement» consenti et même recherché par la femme de Boston et de Tahiti: «J'ai toujours désiré et je désire encore de me marier, et . . j'aurais la bonne conduite, l'industrie et l'économie convenable à une femme, comme j'en ai la fécondité» (Supplément 166). Diderot abhore l'homosexualité féminine (La religieuse) et tout ce qui pourrait être perçu comme contraire à l'harmonie «naturelle» des sexes. Ses vénus tahitiennes et anglaises (et ses «bijoux indiscrets») réclament à grands soupirs et par des prières insistantes le mariage producteur d'enfants (voir le roman de jeunesse de Diderot intitulé Les bijoux indiscrets). Diderot se montre plus réaliste en ce qui 
concerne l'exploitation sexuelle des épouses dans «Sur les femmes». Néanmoins, dans le Supplément, Tahiti se prête aux épousailles et à la spéculation comme Orou, comme Miss Baker. La nature féconde des colonies promet de travailler et de reproduire avec un maximum de rendement pour le bénéfice de l'état policé. Les dialogues du Supplément répondent à une exigeance d'économie coloniale. Le discours du prêtre n'a pas cours. Les exigeances de moralités sont court-circuitées puisque le prêtre doit se prêter à un dialogue mi-philosophique mi-commercial. Ses actes procréateurs disqualifient d'avance un discours catholique.

Cependant, dans le Supplément, il ne s'agit pas vraiment d'établir un commerce, ni même un dialogue avec Tahiti. Contrairement à ce que le discours d'Orou semblait suggérer, le bénéfice commercial reste unilatéral. Il n'est pas même question de convertir, de convaincre les peuples d'adopter la Bible, ni de leur apporter quoi que ce soit comme Lumières: l'aumônier ne cherche pas à faire des prosélytes; les marins de Bougainville détruisent et reproduisent dans un même souffle. Il ne s'agit pas du «logos» défini comme "calcul, raisonnement, parole, discours, connaissance» (Derrida, L'autre cap 97, en italique dans le texte). Il est plutôt question d'une topographie, comme lieu d'énonciation, de transport, de cession.

\section{L'un y gagne ce que l'autre perd}

Car les colonies modernes - de type cinq et six, selon la classification de l'article «COLONIE» de l'Encyclopédie - «enrichissent la métropole», ne sont fondées que "pour l'utilité de la métropole» (Enc. III: 650). Selon ce même article, "COLONIE» est un mot lié à l'histoire ancienne, moderne, et commerciale, et commence avec la mort de Noé dont les descendants «trop multipliés pour habiter ensemble» doivent chercher des ressources séparément (Enc. III: 648). Avec le deuxième type de colonie, l'humanité couvre la terre, jusqu'à ce que l'excès de population nécessite l'établissement de "propriétés distinctes", qui entraîne des "différends» et surtout «l'âge des conquérants», tel Alexandre, dans des colonies de type trois (Enc. III: 649). Aux colonies barbares (type quatre) succèdent les colonies commerciales modernes, dont celles de l'Amérique qui sont aussi de "culture»: «Dès-lors il était nécessaire de conquérir les terres, \& d'en chasser les anciens habitans, pour y en transporter de nouveaux», les surplus de population dont les métropoles devraient surveiller l'exode pour le limiter, au cas où elles en viendraient à se vider de leurs populations essentielles (Enc. III: 650). Les colonies sont directement causées par les mariages fructueux, par l'accroissement excédentaire des familles, dans le but d'accroître un 
supplément de population dévoué et fidèle, qui enrichisse la métropole en soldats, en travailleurs, en denrées et matières multiples.

Le fait que Diderot soit populationniste prend toute sa valeur après la lecture de l'article «COLONIE» dans l'Encyclopédie dont il partageait la direction avec d'Alembert. Hervé Hasquin a situé la pensée diderotienne aux origines du libéralisme économique (non dirigiste) et démocratique $(111,121)$ : «D'un côté, les populationnistes. Ils sont en partie les héritiers des mercantilistes et estiment que la population crée la richesse; par conséquent, tout doit être mis en oeuvre pour l'accroître. En face, les physiocrates, et à leur tête Quesnay; ils renversent le postulat» (117). Selon Hasquin, Diderot ne «sortira jamais» du camp populationniste, contre les physiocrates qui préconisent un accroissement des populations proportionné aux richesses. Quesnay ne rédige pas l'article «HOMME» qui lui était réservé. Diderot s'en charge en 1765, réclamant "plus d'ouvriers, plus de commerçants, plus de soldats", et il récidive en 1772 à l'article «SOUVERAIN» (Hasquin 117-118).

Si Orou diderotien cherche à décrire un contact mutuellement bénéfique, il reste en défaut comme la théorie économique moderne fondée par Condillac - Derrida nous le rappelle après Marx, qui "dénonce une confusion entre la valeur d'usage et la valeur d'échange» en ce philosophe des Lumières (Frivole 86):

Il est faux, dit cet écrivain [Condillac], que, dans les échanges, on donne valeur égale pour valeur égale. Au contraire chacun des contractants en donne toujours une moindre pour une plus grande. ... En effet, si on échangeait toujours valeur égale pour valeur égale, il n'y aurait de gain à faire pour aucun des contractants. Or, tous les deux en font ou en devraient faire. Pourquoi? C'est que les choses n'ayant qu'une valeur relative à nos besoins, ce qui est plus pour l'un est moins pour l'autre, et réciproquement. . . Ce ne sont pas les choses nécessaires à notre consommation que nous sommes censés mettre en vente.... Nous voulons livrer une chose qui nous est inutile, pour nous en procurer une qui nous est nécessaire. (Derrida, Frivole 87, après Marx, Capital 162-63)

Ainsi Orou, Aotourou, poursuivent une chimère de commerce, d'échange, de réciprocité, par rapport à des interlocuteurs opportunistes, à des commerçants avantageusement fautifs. Il s'agit de bénéficier à un seul pôle dans ces échanges qui n'ont pas même valeur de dialogue.

Entre la culture d'Orou et celle de l'aumônier, il existe un constat, une définition de l'Europe. L'Europe, c'est le destin de Tahiti. Le transfert du corps tahitien en Europe n'est pas nécessaire: il s'y trouve déjà fossilisé - l'homme naturel dans l'homme français. Compatibilité de l'utopie ou de la dystopie, Tahiti de Bougainville est le seul pays qui, selon B., lui ait 
inspiré «du goût pour une autre contrée que la [s]ienne» (Supplément 146). Sans doute s'agissait-il finalement de la même contrée. Orou et Diderot partagent les mêmes appétits avides et la même foi en la natalité, bien qu'Orou et le vieillard considèrent la possibilité que la fin de leur culture vienne de l'Europe envahissante. Le tableau optimiste de Diderot projette une ombre. Si Orou et l'aumônier possèdent la même prétention coloniale, comprise dans les mêmes ambitions natalistes, une guerre est possible, non pas entre les pays «civilisés» et leurs colonies, mais entre puissances égales.

Orou indique à l'aumônier qu'il s'agit de couvrir la terre d'une humanité qui en exploite exhaustivement toutes les ressources: «La terre où tu es né a-t-elle plus d'hommes qu'elle n'en peut nourrir? en ce cas tes moeurs ne sont ni pires, ni meilleures que les nôtres. En peut-elle nourrir plus qu'elle n'en a? nos moeurs sont meilleures que les tiennes» (Supplément 154). Orou diderotien prône le principe d'une population excédante aux ressources du territoire. Le natalisme peut aussi donner lieu à un scénario moins optimiste:

B. .. Quelle communication les liait autrefois avec le reste de leur espèce? que deviennent-ils en se multipliant sur un espace qui n'a pas plus d'une lieue de diamètre?

A. Ils s'exterminent et se mangent....

B. Ou la multiplication y est limitée par quelque loi superstitieuse ...

A. Ou l'homme égorgé expire sous le couteau d'un prêtre; ou l'on a recours à la castration des mâles...

B. A l'infibulation des femelles; et de là tant d'usages d'une cruauté nécessaire et bizarre.... (Supplément 144)

C'est là l'antithèse d'une moralité qui condamne à l'exil et à l'esclavage les femmes stériles ou vieillies - inutiles - qui persistent dans leurs relations sexuelles.

\section{Les enjeux}

Qu'ils cultivent ou qu'ils disputent à l'espèce animale, l'activité des nombreux enfants est l'expansion territoriale: «Toute guerre naît d'une prétention commune à la même propriété. L'homme civilisé a une prétention commune, avec l'homme civilisé, à la possession d'un champ dont ils occupent les deux extrémités. . . . Et le tigre a une prétention commune, avec l'homme sauvage, à la possession d'une forêt» (Supplément 145). Tant qu'Orou se fait le porte-parole des valeurs tahitiennes, il y a quelque futilité à dénoncer cette pratique de Bougainville, qui est 
d'enterrer dans tous les lieux qu'il accoste une plaquette exprimant son désir: «ce pays est à nous» (Supplément 148).

Comme les "sauvages» du Moluque, le vieillard tahitien du Supplément s'indigne de cette pratique, parce qu'elle lui est présentement défavorable. Mais les Tahitiens ont fait en sorte que leurs femmes et leur terre nourissent et accueillent les Européens et leurs descendants: «Plus robustes, plus sains que vous, nous nous sommes bien aperçus au premier coup d'oeil que vous nous surpassiez en intelligence; et, sur le champ, nous avons destiné quelques-unes de nos femmes et de nos filles les plus belles à recueillir la semence d'une race meilleure que la nôtre» (Supplément 175). Tahiti fournit la matière, les colons l'esprit. Ainsi, Orou reconnaît (selon les valeurs diderotiennes) que Tahiti doit être enrichie de la présence française, à l'intellect génétiquement supérieur. Les Tahitiens cèdent leurs possessions, femmes et champs, qui permettent l'implantation coloniale. Au fait, c'est bien "sur le champ» que les Tahitiens de Diderot ont donné leur place et cédé du terrain. Il n'y a donc pas d'antithétie, de guerre déclarée entre Tahiti et la France, si ce n'est par une génération finissante, effrayée par l'évolution moderne de sa société et qui plaide brutalement pour un retour en arrière. Les «lâches ennemis» benéficient d'un accueil amical, curieux, d'êtres que cherchent à connaître. Au contraire de ce qu'Orou estime, ce sont les Français qui sont en fait intéressés par la matière. Ils veulent manger, consommer, s'approprier, là où ils rencontrent une curiosité intellectuelle, des civilités confiantes, une douceur de moeurs.

Ainsi, lorsque Bougainville enregistre les ressources d'un lieu et le degré de présence humaine, d'hospitalité, il poursuit la même politique $d^{\prime}$ enrichissement en population et en espace vital qu'Orou. La possession commune des femmes et de la nourriture entraîne à Tahiti la suppression des frontières internes, mais aussi de façon unilatérale en ce qui concerne les frontières externes de Tahiti, qui tombent paisiblement par mariage plutôt que par les armes pour le bénéfice de l'expansion du territoire attaché à la couronne de France. C'est sans doute ici qu'est le lieu stratégique de l'énoncé diderotien, qui le met dans le camp de la colonisation et de la propriété territoriale - lieu commun de l'humanité et du tigre?

\section{Portrait du colonisateur en l'épouser du genre humain?}

Dom Juan de Molière a les principes d'Orou: «Toutes les belles ont droit de nous charmer, et l'avantage d'être rencontrée la première ne doit point dérober aux autres les justes prétentions qu'elles ont toutes sur nos coeurs» (Molière 360). Il est abondamment question du mariage dans le 
Supplément. A. demande à B. si le mariage «est dans la nature» et comprend qu' «il se forme une union plus ou moins durable, qui perpétue l'espèce par la reproduction des individus» (Supplément 179). ${ }^{6} \mathrm{Ce}$ type de mariage est conçu d'abord dans le cadre tahitien comme instrument de colonisation. Bougainville et son équipage, aussi bien que B., se sentiraient «du goût» pour Tahiti à la manière de dom Juan, «l'épouseur du genre humain» (Supplément 146; Molière 378): «Je me sens un coeur à aimer toute la terre; et comme Alexandre, je souhaiterais qu'il y eût d'autres mondes, pour y pouvoir étendre mes conquêtes amoureuses» (Molière 361). Et quand il s'agit de faire parler cet autre insondable, tahitien ou féminin, c'est sans doute par le truchement des lèvres de la génération qu'il faut accéder à une vérité de fantaisie magistrale, faire fructifier Les bijoux indiscrets.

Otto Rank précise:

La situation de Don Juan vis-à-vis du mari [est] celle d'un être supérieur ... du dieu [et] vis-à-vis l'homme . . q qu'il ne veut pas l'évincer ou l'écarter, mais l'emporter sur lui, soit par la ruse, soit par le vol . . . une forme tardive de l'ancienne croyance à l'âme par laquelle l'homme fort et volontaire a le droit et le devoir de féconder les femmes pour perpétuer son âme. (158)

L'économie de dom Juan selon Otto Rank est celle du "prélèvement» (Rank 158). Le héros de la colonisation du Supplément au voyage de Bougainville réconcilie deux philosophies contradictoires de la chrétienté, en celle d'un prêtre qui engendre des âmes. Ce héros à la fois païen et chrétien est synthétisé par l'aumônier procréateur à Tahiti:

Don Juan est encore l'antique héros qui féconde les âmes, mais sous l'influence de l'idée chrétienne du péché, il est devenu un jouisseur sexuel, dévergondé. Pour mieux dire, il aurait failli le devenir si la croyance en une immortalité personnelle, comme elle existait avant l'idéologie chrétienne, ne s'était pas défendue contre une immortalité trop spiritualisée et trop communiste telle que l'enseigne la religion chrétienne. (Rank 159)

Comme les marins de Bougainville, il opère dans le cadre du mariage des Tahitiennes à des Tahitiens, avec une valorisation extrême des enfants coloniaux, qui par leur expansion, leur implantation en sol étranger bénéficieraient à l'humanité tout entière, de l'aveu des colonisés. Il s'agit aussi de créer un surplus de population enfantée par la France, sans que le territoire français ne perde aucun de ses sujets. 


\section{Notes}

1 Le but de cet article n'est pas d'interdire qu'une nation soit représentée par un être qui lui est externe et qui n'est pas légitimement élu par cette nation pour la représenter. Un porte-parole auto-désigné peut dire et faire autant de bien que de mal qu'un représentant élu le plus démocratiquement du monde. Il suffit de considérer Poulain de la Barre et Stuart-Mill qui ont fort bien parlé de leur propre initiative pour les femmes.

2 Dans son excellent article sur le Supplément comme «une poétique du déguisement», Marie-Hélène Chabut remarque la «masculinisation de la notion d'individu» et l'inclusion des femmes dans la catégorie des possessions communes (13). Elle exploite le symbolisme du déguisement de Mlle Barré, qui peut fonctionner comme un homme dans la société française - pourvu qu'elle adopte le vêtement masculin — , mais qui est immédiatement identifiée comme objet de propriété sur les rivages de l'île, où les Tahitiens «ne reconnaissent pas leur image» en elle (13). Son article relève les contradictions majeures à l'intérieur $\mathrm{du}$ discours d'Orou qui critique dans la culture de l'aumônier ce qui se pratique tout pareillement dans l'île.

3 Il existe un traitement uniforme des "curiosités» étrangères, des petits singes (tamarins) du salon de la Pompadour, à la «Venus hottentote» stéatopyge montrée dans les divers salons et théâtres européens. Ils finissent aussi souvent sous le scalpel.

4 Whatley analyse brillamment l'aspect politique et social de l'amour («Un retour secret»). Son article fait pendant à la pensée de Perkins. Kra parle aussi du rôle des relations sexuelles dans la pensée de Montesquieu («The Role of the Harem»).

5 Crampe-Casnabet remarque que «comme Montesquieu, Condorcet soutient qu'un nègre libre aura plus d'intérêt au travail» (55). La même argumentation fonctionnerait pour les serviteurs et les femmes, attachés au maître par un amour familial. Selon Crampe-Casnabet, Montesquieu diffèrerait de Jaucourt, auteur de l'article «ESCLAVAGE» de l'Encyclopédie, parce qu'il contesterait la possibilité de confondre la servitude et le salariat. Néanmoins, cet amour inconditionnel du travail et des maîtres, que nous retrouvons encore dans la pièce d'Olympe de Gouges introduite par ses "Réflexions sur les hommes nègres» relie aussi la question de l'esclavage, des serviteurs, des femmes, des sujets, qui tous n'ont que leur vertueuse obéissance pour se défendre - ou la rébellion criminelle.

6 Benrekassa résume Duchet qui donne une explication du Supplément: «On nous dévoile comment le 'thème' du bonheur de l'état sauvage et du malheur de l'homme civilisé, celui de la liberté sexuelle ou de la condition des femmes, le problème des trois codes, de leur conformité ou de leur discordance, celui du maintien de l'homme naturel dans l'homme social, celui de la nécessité relative de maintenir un ordre, sont au centre des réflexions de Diderot dans un nombre d'écrits contemporains»; et «on tente de nous montrer en même temps que la conception que Diderot se fait du rapport entre la loi civile et la loi naturelle l'amène à tenir pour vain d'accuser 'les moeurs de l'Europe par celles d'Otaïti'; ... mais que d'autre part Tahiti fonctionne comme un 'mythe personnel' et même comme un 'mythe politique' . . . une invitation à en appeler toujours à la 'Nature'» $(215,216)$. Les contradictions relevées par Benrekassa se résument en ce que Diderot est inconditionnellement obéissant au statu quo, à la manière d'Iñes de Castro, personnage d'Houdar de la Motte, dont la vertu sacrificielle consiste essentiellement en l'obéissance aux maîtres (époux, père ou roi). 


\section{Textes cités}

Abbeele, George Van Den. «Utopian Sexuality and its Discontents: Exoticism and Colonialism in Le Supplément au voyage de Bougainville». L'esprit créateur 24 (1984): 4352.

Adam, Antoine. «Introduction». Diderot, Pensées. 15-26.

Alembert, Jean Le Rond d', et Denis Diderot. Encyclopédie ou dictionnaire raisonné des sciences, des arts et des métiers. Facsimilé. 35 vols. Neufchastel: Samuel Faulche \& Compagnie, 1751-1780. Rpt Stuttgart-Bad Cannstatt: Friedrich Frommann Verlag, 1966.

Benrekassa. Georges. Le concentrique et l'excentrique: marges des Lumières. Paris: Payot, 1980.

Bougainville, Louis-Antoine de. Voyage autour du monde par la frégate du Roi, La Boudeuse et la flûte L'étoile. Ed. Jacques Proust. Paris: Gallimard, 1982.

Chabut, Marie-Hélène. «Le Supplément au voyage de Bougainville: une poétique du déguisement.» Diderot Studies 24 (1991): 11-23.

Crampe-Casnabet, Michèle. Condorcet lecteur des Lumières. Paris: PUF, 1985.

Derrida, Jacques. L'archéologie du frivole: lire Condillac. Paris: Éditions Galilée, 1973. L'autre cap. Paris: Les Éditions de Minuit, 1991.

Diderot, Denis. Pensées philosophiques, addition aux Pensées philosophiques, Lettres sur les aveugles, addition aux Lettres sur les aveugles, Supplément au voyage de Bougainville. Paris: Garnier-Flammarion, 1972.

Grafigny, Françoise de. «Introduction historique aux Lettres péruviennes». Lettres portugaises, Lettres d'une Péruvienne et autres romans d'amour par lettres, 1747. Paris: Garnier Flammarion, 1983. 251-56.

Hasquin, Hervé. «Politique, économie et démographie chez Diderot: aux origines du libéralisme économique démocratique.» Thèmes et figures $d u$ siècle des Lumières. Ed. Raymond Trousson. Genève: Droz, 1980. 107-122.

Kra, Pauline. «The Role of the Harem in Imitations of Montesquieu's Lettres persanes». Studies in Voltaire and the Eighteenth Century 182 (1979): 273-283.

Molière, Jean-Baptiste Poquelin. Dom Juan ou le festin de pierre. Oeuvres complètes. Ed. Georges Mongrédien. Paris: Garnier-Flammarion, 1965. 357-408.

Perkins, Merle Louis. «Community Planning in Diderot's Supplément au voyage de Bougainville». Kentucky Romance Quarterly 21.4 (1974): 399-417.

Rank, Otto. Don Juan et le double: études psychanalytiques. Paris: Payot, 1932, 1973.

Said, Edward W. Orientalism. New York: Vintage Books, 1979.

Whatley, Janet S. «Un retour secret vers la forêt: The Problem of Privacy and Order in Diderot's Tahiti». Kentucky Romance Quarterly 24.2 (1977): 199-208. 